\title{
Effect of non-random mating on genomic and BLUP selection schemes
}

\author{
Kahsay G Nirea ${ }^{1 *}$, Anna K Sonesson², John A Woolliams ${ }^{1,3}$ and Theo HE Meuwissen ${ }^{1}$
}

\begin{abstract}
Background: The risk of long-term unequal contribution of mating pairs to the gene pool is that deleterious recessive genes can be expressed. Such consequences could be alleviated by appropriately designing and optimizing breeding schemes i.e. by improving selection and mating procedures.

Methods: We studied the effect of mating designs, random, minimum coancestry and minimum covariance of ancestral contributions on rate of inbreeding and genetic gain for schemes with different information sources, i.e. sib test or own performance records, different genetic evaluation methods, i.e. BLUP or genomic selection, and different family structures, i.e. factorial or pair-wise.

Results: Results showed that substantial differences in rates of inbreeding due to mating design were present under schemes with a pair-wise family structure, for which minimum coancestry turned out to be more effective to generate lower rates of inbreeding. Specifically, substantial reductions in rates of inbreeding were observed in schemes using sib test records and BLUP evaluation. However, with a factorial family structure, differences in rates of inbreeding due mating designs were minor. Moreover, non-random mating had only a small effect in breeding schemes that used genomic evaluation, regardless of the information source.
\end{abstract}

Conclusions: It was concluded that minimum coancestry remains an efficient mating design when BLUP is used for genetic evaluation or when the size of the population is small, whereas the effect of non-random mating is smaller in schemes using genomic evaluation.

\section{Background}

Selection and parent mating patterns are the two major components of a breeding program and must be optimised with respect to genetic gain $(\Delta \mathrm{G})$ and rate of inbreeding $(\Delta \mathrm{F})$. In the literature, several selection and mating designs have been reported that aim at reaching a high $\Delta \mathrm{G}$ and/or low $\Delta \mathrm{F}[1,2]$, since a high $\Delta \mathrm{F}$ represents a risk for the long-term success of breeding programs. While there is a consensus on selection procedures [3,4], many mating designs have been developed, mostly aimed at avoiding the mating of parents that are more related than average [1,5-7].

Factorial mating consists in producing half sibs from male and female parents at the expense of full sibs, while preserving offspring numbers per parent [8]; with minimum coancestry mating $(\mathrm{MC})$, relationships

\footnotetext{
* Correspondence: kahsay.nirea@umb.no

'Department of Animal and Aquacultural Sciences, Norwegian University of Life Science, Norwegian, P.O. Box 5003 N-1432 Ås, Norway

Full list of author information is available at the end of the article
}

between mating pairs are minimized to minimize progeny inbreeding [9]; with compensatory mating, parents with a small number of selected sibs are preferably mated to those with a large number of selected sibs [10]. More recently, Henryon et al. [2] considered mating animals by minimizing the covariance between ancestral contributions (MCAC), which allows the impact of future changes in ancestral contributions to be less mutually dependent and favours the selection of animals with optimum contributions from their ancestors. All these mating schemes have shown some benefits in increased genetic gain and/or reduced inbreeding but comparisons reported in the literature have not concluded on any clearly superior mating design, partly because only a limited number of mating designs were considered in each of the comparisons.

All studies mentioned above have used evaluation methodologies that rely on phenotype alone or combined with pedigree to provide best linear unbiased prediction (BLUP) estimated breeding values (EBV).

\section{Biomed Central}


Recently, the concept of genomic evaluation has been introduced in animal breeding [11], in which the prediction of the total genetic value of selection candidates is based on dense genotyping data and estimates of SNP effects, which are calculated from a genotyped and phenotyped training set of individuals from the population under selection. One of the reported benefits of this approach is that the accuracy of EBV increases thanks to an improved prediction of the Mendelian sampling term. The consequence is a potential reduction of $\Delta \mathrm{F}$ in selection schemes [12] because it reduces the emphasis put on family information and increases that on the individual's own merit. Hence, it is time to re-examine the benefits of non-random mating given the methodological developments in genomic selection (GS).

Therefore, this study aims to assess the interaction between genetic evaluation methodology and non-random mating designs and their impact on $\Delta \mathrm{F}$ and $\Delta \mathrm{G}$. It compares the impacts of pair-wise versus factorial family structures and performance-testing versus sib-testing, which differ in their information source, the candidate and sibs of the candidate, respectively. Such structure and testing designs are common variants in aquaculture breeding schemes.

\section{Methods \\ Populations}

We simulated a Fisher-Wright population with an effective population size of 1000 and 4000 generations to construct a base generation G0 for use in subsequent selection. The population had a diploid genome with a total length of 10 Morgan with 10 chromosomes, each 1 Morgan long. SNP mutations were introduced in all generations used to generate $\mathrm{G} 0$ at a rate of $10^{-8}$ per base pair per meiosis, assuming

1000000 base pairs per cM. Parent to offspring transmission of SNP followed Mendelian inheritance. A period of 4000 generations has been shown to be sufficient to achieve mutation-drift equilibrium [13]. In each of the 4000 generations, $n_{m}=500$ males and $n_{f}=500$ females were produced by random selection (with replacement) of a male and a female parent. The same procedure was repeated 100 times to create 100 replicates.

In G0, 200 male and 200 female candidates were randomly sampled, i.e. $\mathrm{n}_{\mathrm{m}}=\mathrm{n}_{\mathrm{f}}=200$, and, if appropriate, another sample of identical size was randomly chosen as test sibs. From G0 until G12, $\mathrm{n}_{\mathrm{s}}$ sires and $\mathrm{n}_{\mathrm{d}}$ dams were selected and mated according to the procedures described below. Each mating produced $2 \mathrm{n}_{\mathrm{o}}$ offspring per mating pair, equally divided between males and females, where $\mathrm{n}_{\mathrm{s}}=\mathrm{n}_{\mathrm{d}}=25$ (or 50 ), with $2 \mathrm{n}_{\mathrm{o}}=2,8$ or 16 depending on the family structure (see below). The total number of candidates in each generation G1 to G12 was 400, equally divided between males and females, with an additional 400 if required for sib testing. In aquaculture breeding programs, the fixed cost of conducting a test is an important resource constraint, rather than the variable cost of producing additional sibs for testing, if testing is necessary.

\section{Genome, markers and true breeding values}

In G0, a random sample of 1000 SNP with minor allele frequency $(\mathrm{MAF})>0.05$ were considered as quantitative trait loci (QTL). Allelic values were assigned to the QTL by independent sampling of effects from the Laplace distribution. The QTL effects were assumed to be additive and effects were standardized such that the total genetic variance (variance of breeding values) was 10 . Phenotypes were also created assuming a heritability of 0.4 or 1 . If the heritability was 1 , the phenotype was equal to the true breeding value but if it was 0.4 , an environmental deviation was drawn independently from $\mathrm{N}(0,15)$ and added to the true breeding value for G0 to G12. Our objective was to track $m=5000$ markers from G0 onwards and since more SNP were generated than needed SNP with the highest MAF were selected from those not selected as QTL.

\section{Breeding schemes: Recording, estimation of breeding values, selection and mating Information source}

We studied the effect of selection and mating on $\Delta \mathrm{F}$ and $\Delta G$ with either a performance test of the candidates (CAND) or a sib test (SIB). In the CAND schemes, the candidates were directly recorded for the trait under selection. In the SIB schemes, the full sib families were divided into two sets, i.e. one set was recorded for the trait and one set provided the candidates for selection. This is the case if the trait is for instance a disease challenge or a meat quality assessment resulting in the death of the tested individuals.

\section{Evaluation and selection}

Selection among the candidates was performed by truncation selection of $n_{s}$ sires and $n_{d}$ dams based on the EBV provided by genetic evaluation. Two types of genetic evaluation were considered: (1) BLUP, based on pedigree and phenotypes; and (2) GS using a set of 5 000 SNP, which were assumed to have been genotyped on all individuals of a given generation. BLUP evaluations were done according to the standard methodology of Henderson [14]. Genomic evaluation followed the GS-BLUP model [11] for $\mathrm{n}$ phenotypes with $m$ SNP loci:

$$
\mathbf{y}=\sum_{\mathrm{j}=1}^{m} \mathbf{x}_{\mathrm{ij}} \mathrm{g}_{\mathrm{j}}+\mathbf{e},
$$

where $y$ is a vector of phenotypes, $X_{i j}$ is the standardized number of a reference allele (allele " 1 ") for animal $i$ and SNP $j$ as assessed by SNP i.e. 


$$
\mathrm{x}_{\mathrm{ij}}=\frac{\mathrm{x}_{\mathrm{ij}}^{*}-2 \mathrm{p}_{\mathrm{j}}}{\sqrt{2 \mathrm{p}_{\mathrm{j}}\left(1-\mathrm{p}_{\mathrm{j}}\right)}},
$$

where $P_{j}$ is the frequency of allele " 1 " at SNP $\mathrm{j}, x^{*}{ }_{i j}$ is the number of alleles " 1 " genotyped for animal $i$ at $\mathrm{SNP}_{\mathrm{ij}}, g_{\mathrm{j}}$ is the effect of allele " 1 " at locus $\mathrm{j}$, and $e$ is the vector of random errors assumed to be drawn from $\mathrm{N}$. The elements $\mathrm{x}_{\mathrm{ij}}$ form the incidence matrix $\mathbf{X}$. The SNP effects were estimated as

$$
\left[\mathbf{X}^{\prime} * \mathbf{X}+\lambda * \mathbf{I}\right][\hat{\mathbf{g}}]=\left[\mathbf{X}^{\prime} * \mathbf{y}\right] .
$$

From the above mixed model equation $g$ is the vector of estimated effects at the marker loci, and $\lambda=m \frac{\sigma_{\mathrm{e}}^{2}}{\sigma_{\mathrm{A}}^{2}}$. The variance of each marker effect was assumed to be $\sigma_{i}^{2}=\frac{\sigma_{A}^{2}}{m}$.

Finally, the genomic EBV $(\hat{\boldsymbol{u}})$ for selection candidate i was estimated as

$$
\hat{u}_{i}=\sum_{\mathrm{j}=1}^{m} \mathrm{x}_{\mathrm{ij}} \hat{\mathrm{g}}_{\mathrm{j}}
$$

\section{Family structure}

Two family structures were studied:

(1) The first was a pair-wise family structure (PAIR), where identical numbers of sires and dams, i.e. $n_{s}=n_{d}$ $=25$ (or 50), were selected every generation and each sire was only mated to one dam, to produce 25 (or 50 ) full-sib families. Each full-sib family had $2 \mathrm{n}_{\mathrm{o}}=16$ (or 8 ) offspring, with an equal sex ratio. Thus, a total of 400 candidates were produced every generation. For the SIB schemes, the $2 n_{o}$ offspring were doubled to produce test sibs

(2) The second structure was a factorial family structure (FAC), where identical numbers of sires and dams, i.e. $\mathrm{n}_{\mathrm{s}}=\mathrm{n}_{\mathrm{d}}=25$ (or 50 ), were selected every generation and mated so that each sire was mated with 8 (or 4 ) dams and each dam was mated with 8 (or 4 ) sires, producing 200 full-sib families, with $2 \mathrm{n}_{\mathrm{o}}=2$, with 25 (or 50) paternal half- sib families and 25 (or 50) maternal half-sib families.

\section{Mating design}

Three mating designs were studied:

(1) Random mating (RAND), in which selected sires and dams were paired by random sampling without replacement from the candidates

(2) Minimum coancestry mating (MC), where the sets of male and female mating pairs were chosen in order to minimize the average coancestry between the mates, as calculated from the pedigree. Randomly-chosen parents were swapped until a decrease in coancestry was observed, at which point the swapped parents were retained, as in [5]. This was repeated until no more improvement was achieved.

(3) Minimized covariance of ancestral contributions mating (MCAC), in which contributions of each ancestor to each candidate were calculated and the covariance between the contributions for the proposed set of male and female mating pairs was minimised [2]. Starting from the random mating design, parents were randomly chosen and swapped until the MCAC mating criterion improved and then the swapped parents were retained. The process was stopped when no further improvement of MCAC was achieved.

\section{Simulated schemes}

The three mating designs (RAND, MC and MCAC) were tested for all combinations of evaluations (BLUP or GS), family structures (PAIR or FAC), information sources (CAND or SIB), and $h^{2}(0.4$ or 1$)$. This amounts to a total of 48 comparisons. An additional comparison was made considering an increased number of parents $\left(\mathrm{n}_{\mathrm{s}}\right.$ and $\left.\mathrm{n}_{\mathrm{d}}\right)$.

\section{Statistics for assessment}

For each scheme, $\Delta \mathrm{G}$ and $\Delta \mathrm{F}$ were calculated. $\Delta \mathrm{F}$ was computed as the negative of the slope of the regression of $\ln \left(1-\mathrm{F}_{\mathrm{t}}\right)$ on $\mathrm{t}$ for $\mathrm{F}_{6}-\mathrm{F}_{12}$ where $\mathrm{F}_{t}$ is the level of inbreeding at generation $t, \Delta \mathrm{G}$ was computed as the difference in genetic level between G12 and G6 divided by 6 . The use of generations 6 to 12 made it possible to reach a near-Bulmer equilibrium and to stabilize the degree of assortative mating. Each simulation was replicated 100 times and the replicates were averaged. Standard errors of the mean were calculated from the variance between replicates.

\section{Results}

Tables 1, 2 and 3 present rates of inbreeding $(\Delta \mathrm{F})$ and genetic gain $(\Delta G)$ generated under different evaluation methods (GS or BLUP), information sources (CAND or SIB), family structures (FAC or PAIR), and mating designs (RAND, MC and MCAC) for discrete generations with an initial heritability of 0.4 or 1 . Figure 1 shows a summary of the results.

\section{Effect of genetic evaluation methods}

The effect of the genetic evaluation method was obtained by comparing the $\Delta \mathrm{F}$ and $\Delta \mathrm{G}$ generated under GS/CAND and BLUP/CAND, GS/SIB and GS/CAND schemes (Table 1) using the RAND mating design. For PAIR family structures, a $0.016(40 \%)$ reduction in $\Delta \mathrm{F}$ and a $0.096(14 \%)$ increase in $\Delta \mathrm{G}$ were observed when 
Table 1 Effect of mating when family structure, information source and genetic evaluation vary and $h^{2}=$ 0.4

\begin{tabular}{llcccc}
\hline Scheme & Mating & \multicolumn{2}{c}{$\begin{array}{c}\text { Pair-wise family } \\
\text { structure }\end{array}$} & \multicolumn{2}{c}{$\begin{array}{c}\text { Factorial family } \\
\text { structure }\end{array}$} \\
\cline { 3 - 6 } & & $\Delta \mathrm{F}$ & $\Delta \mathrm{G}$ & $\Delta \mathbf{F}$ & $\Delta \mathbf{G}$ \\
\hline BLUP/CAND & RAND & 4.09 & 0.684 & 2.02 & 0.731 \\
\hline & MC & 1.98 & 0.723 & 2.02 & 0.766 \\
\hline & MCAC & 3.41 & 0.741 & 1.84 & 0.741 \\
\hline BLUP/SIB & RAND & 8.95 & 0.460 & 3.34 & 0.581 \\
\hline & MC & 3.47 & 0.522 & 3.13 & 0.589 \\
\hline & MCAC & 6.45 & 0.504 & 2.98 & 0.580 \\
\hline GS/CAND & RAND & 2.45 & 0.780 & 1.58 & 0.829 \\
\hline & MC & 1.52 & 0.814 & 1.55 & 0.813 \\
\hline & MCAC & 2.39 & 0.813 & 1.49 & 0.841 \\
\hline GS/SIB & RAND & 2.67 & 0.751 & 1.64 & 0.797 \\
\hline & MC & 1.54 & 0.777 & 1.53 & 0.801 \\
\hline & MCAC & 2.52 & 0.776 & 1.57 & 0.785 \\
\hline
\end{tabular}

Rates of inbreeding in percentage $(\Delta \mathrm{F})$ and genetic gain in genetic standard deviation units $(\Delta G)$ generated per generation when $n_{s}=25, n_{d}=25$ and $h^{2}$ $=0.4$ for schemes where parent selection is on BLUP EBV using candidate information (BLUP/CAND) or using sib information (BLUP/SIB); for schemes where parent selection is on GS EBV using candidate information (GS/CAND) or sib information (GS/SIB); RAND: random; MC: minimum coancestry; MCAC: minimizing covariance of ancestral contributions mating; standard errors of means of 100 replicates of genetic gain were less than $0.38 \%$ and of rates of inbreeding were less than $0.11 \%$

candidate records were used for GS evaluation compared to BLUP evaluation. There was a $0.063(70 \%)$ reduction in $\Delta \mathrm{F}$ and a $0.291(60 \%)$ increase in $\Delta \mathrm{G}$ when sibs of the candidates were recorded for genetic evaluation (Table 1). For FAC family structures, a $0.004(9 \%)$ reduction in $\Delta \mathrm{F}$ and a $0.098(13 \%)$ increase in $\Delta \mathrm{G}$ were observed when the candidates were recorded and a $0.017(51 \%)$ reduction in $\Delta \mathrm{F}$ and a $0.216(37 \%)$ increase

Table 2 Effect of mating when family structure and genetic evaluation vary and $h^{2}=1$

\begin{tabular}{llcccc}
\hline Scheme & Mating & \multicolumn{2}{c}{$\begin{array}{c}\text { Pair-wise family } \\
\text { structure }\end{array}$} & \multicolumn{2}{c}{$\begin{array}{c}\text { Factorial family } \\
\text { structure }\end{array}$} \\
\cline { 3 - 6 } & & $\boldsymbol{\Delta} \mathbf{F}$ & $\boldsymbol{\Delta G}$ & $\Delta \mathrm{F}$ & $\Delta \mathbf{G}$ \\
\hline BLUP/SIB & RAND & 1.95 & 0.885 & 1.37 & 0.939 \\
\hline & MC & 1.32 & 0.985 & 1.45 & 0.985 \\
\hline GS/SIB & RAND & 1.90 & 0.894 & 1.36 & 0.928 \\
\hline \multirow{2}{*}{ MCAC } & 2.07 & 0.945 & 1.28 & 0.909 \\
\hline & MC & 1.33 & 0.928 & 1.33 & 0.950 \\
\hline & MCAC & 2.04 & 0.916 & 1.29 & 0.951
\end{tabular}

Rates of inbreeding in percentage $(\Delta \mathrm{F})$ and genetic gain in genetic standard deviation units $(\Delta G)$ generated per generation when $n_{s}=25, n_{d}=25$ and $h^{2}$ $=1$ for schemes where parent selection is on BLUP EBV using sib information (BLUP/SIB); for schemes where parent selection is on GS EBV using sib information (GS/SIB); RAND: random; MC: minimum coancestry; MCAC: minimizing covariance of ancestral contributions mating; standard errors of means of 100 replicates of genetic gain were less than $0.21 \%$ and of rates of inbreeding were less than $0.38 \%$
Table 3 Effect of mating when selection intensity changes with a pair-wise family structure

\begin{tabular}{llll}
\hline Scheme & Mating & $\boldsymbol{\Delta} \mathbf{F}$ & $\boldsymbol{\Delta} \mathbf{G}$ \\
\hline BLUP/SIB & RAND & 0.0123 & 0.494 \\
\hline & MC & 0.0114 & 0.496 \\
\hline GS/SIB & MCAC & 0.0106 & 0.488 \\
\hline & RAND & 0.0068 & 0.648 \\
\hline & MC & 0.0060 & 0.659 \\
\hline & MCAC & 0.0038 & 0.646
\end{tabular}

Rates of inbreeding in percentage $(\Delta \mathrm{F})$ and genetic gain in standard deviation units $(\Delta G)$ generated per generation when $n_{s}=50, n_{d}=50$ and $h^{2}=0.4$; standard errors of means of 100 replicates of genetic gain were less than $0.27 \%$ and of rates of inbreeding were less than $0.1 \%$

in $\Delta \mathrm{G}$ when sibs of the candidates were recorded (Table 1). The pattern was similar when the intensity of selection and heritability were increased (Results not shown). Similar results were reported in $[13,15]$, in which sib records were used for BLUP and GS evaluation.

\section{Effect of information sources}

The effect of information sources for schemes with BLUP and GS evaluation is presented in Table 1. The results show that non-random mating designs reduced $\Delta \mathrm{F}$ by a greater factor for schemes with SIB information than for those with CAND information. For example, $M C$ had a lower $\triangle F$ than RAND under the BLUP/ CAND (0.021 i.e. $52 \%$ higher) and the BLUP/SIB (0.055 i.e. $61 \%)$ schemes. Similarly, MCAC had a lower $\triangle \mathrm{F}$ than RAND under the BLUP/CAND (0.007 i.e. 17\%) and the BLUP/SIB (0.025 i.e. 28\%) schemes. Hence, these results show that a larger reduction in $\triangle \mathrm{F}$ was generated by $\mathrm{MC}$ mating compared to MCAC mating.

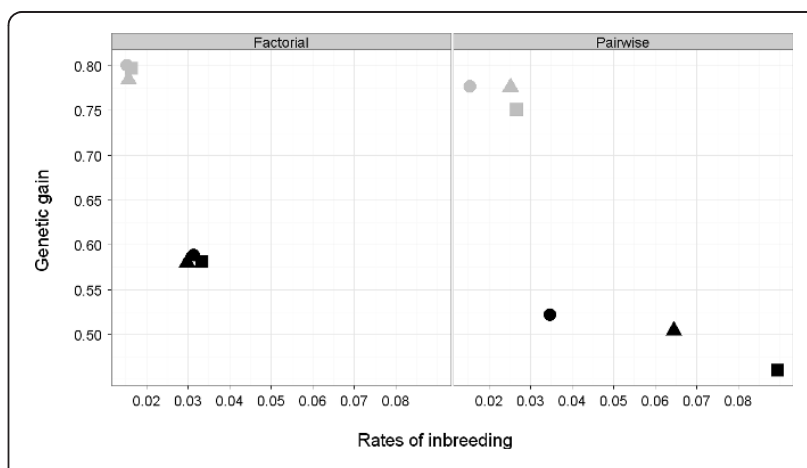

Figure 1 Interactions between genetic gain and rates of inbreeding in different scenarios. RAND or random mating $(\square)$, $M C$ or minimum coancestry $(0)$ and $M C A C$ or minimizing covariance of ancestral contributions mating $(\Delta)$ mating designs with black color for the BLUP/SIB scheme: where $n_{s}=25$ and $n_{d}=$ 25 parents were selected on BLUP EBV using sib information; with grey color for the GS/SIB schemes: where $n_{s}=25$ and $n_{d}=25$ parents were selected on GW EBV using sib information; the family structure of the breeding schemes was either factorial (FAC) or pair wise (PAIR), $h^{2}=0.4$. 
$\Delta G$ was always higher in schemes with CAND information compared to schemes with SIB information and $M C$ and MCAC had a higher $\triangle G$ than RAND. For example, $M C$ had a higher $\triangle G$ than RAND for the BLUP/CAND (0.057 i.e. 6\%) and the BLUP/SIB (0.062 i. e. $13 \%)$ schemes; MCAC also had a higher $\triangle \mathrm{G}$ than RAND for the BLUP/CAND (0.057 i.e. $8 \%$ ) and the BLUP/SIB (0.044 i.e. 10\%) schemes.

The differences in $\Delta \mathrm{F}$ between schemes with GS evaluation were smaller than those with BLUP evaluation, irrespective of the information source. As shown in Table $1, M C$ and MCAC had a lower $\triangle F$ than RAND for the GS/CAND (respectively, 0.009 i.e. $38 \%$ and 0.001 i.e. $2 \%$ ) and the GS/SIB schemes (respectively, 0.011 i.e. $42 \%$ and 0.002 i.e. $6 \%$ ).

$\mathrm{MC}$ and MCAC generated somewhat higher $\triangle \mathrm{G}$ than RAND: 0.033 i.e. $4 \%$ for the GS/CAND scheme and 0.025 i.e. $3 \%$ for the GS/SIB scheme. $\Delta G$ was always higher in the schemes with CAND information than in schemes with SIB information. However, compared to the $\Delta G$ generated by RAND, higher rates of $\Delta G$ were observed by MC and MCAC, especially in the schemes with SIB information. These results show that mating designs such as $M C$ and MCAC can generate higher $\triangle G$ when BLUP evaluation uses sib information compared to candidate information.

In general, compared to schemes with BLUP evaluation the effect of information source (CAND or SIB) was smaller in schemes with GS evaluation (Table 1).

\section{Effect of family structures}

The impact of family structure (PAIR or FAC) on $\Delta \mathrm{F}$ and $\Delta \mathrm{G}$ was assessed by comparing schemes with BLUP evaluation in Table 1. The effect of MC and MCAC on $\Delta \mathrm{F}$ was larger in the schemes with a PAIR family structure than in those with a FAC family structure. For instance, under the BLUP/SIB scheme, MC and MCAC had a lower $\Delta \mathrm{F}$ (respectively, 0.055 i.e. $61 \% 0.025$ i.e. $28 \%)$ than RAND in schemes with a PAIR structure; whereas, in schemes with a FAC structure, MC and RAND had a similar $\triangle \mathrm{F}$ and MCAC had a lower $\triangle \mathrm{F}$ (0.002 i.e. 6\%). Likewise, MC and MCAC had a higher $\triangle \mathrm{G}$ than RAND in schemes with a PAIR structure (respectively, 0.062 i.e. $13 \%$ and 0.44 i.e. $10 \%$ ) but in schemes with a FAC structure, MC had a higher $\triangle G$ than RAND (0.008 i.e. 1\%) and MCAC had a similar $\Delta \mathrm{G}$.

The impact of family structure on $\Delta \mathrm{F}$ for schemes with GS evaluation was evaluated by comparing schemes with GS evaluation in Table 1. In non-random mating designs, $\Delta \mathrm{F}$ was lower for schemes with a FAC structure than for schemes with a PAIR structure. For instance, comparisons for the GS/SIB schemes shows that MC and MCAC led to a lower $\triangle F$ than RAND in the schemes with a PAIR structure (respectively, 0.011 i.e. $42 \%$ and 0.002 i.e. $6 \%$ ) and with a FAC structure (respectively, 0.001 i.e. $7 \%$ and 0.001 i.e. $4 \%$ ). Similarly, comparisons for the GS/SIB schemes show that MC and MCAC had a higher $\triangle G$ than RAND in the scheme with a PAIR structure (respectively, 0.026 i.e. $3 \%$ and 0.025 i.e. $3 \%)$ but in the scheme with a FAC structure, MC had a higher $\triangle \mathrm{G}(0.004$ i.e. $1 \%)$, while MCAC had a lower $\Delta G(0.012$ i.e. $2 \%)$. Similar contrasts were observed for the BLUP/CAND and the GS/CAND schemes. Thus, these results show that the mating designs consistently reduced $\Delta \mathrm{F}$ and increased $\Delta \mathrm{G}$ more for schemes with a PAIR structure than for schemes with a FAC structure. In addition, for schemes with a PAIR structure, the effect of mating design was higher with BLUP evaluation than with GS evaluation. Also, the effect of mating designs decreased when the selection intensity decreased (see Tables 1, 2 and 3).

\section{Effect of heritability}

The results indicate that increasing heritability generally slightly decreased $\Delta \mathrm{F}$ but substantially increased $\Delta \mathrm{G}$ for both family structures. For example, when heritability was increased from 0.4 to 1 for the BLUP/SIB schemes with a PAIR family structure, RAND, MC and MCAC had a lower $\Delta \mathrm{F}$ (respectively, 0.070 i.e. $78 \%, 0.022$ i.e. $62 \%$ and 0.044 i.e. $68 \%$ ) and a higher $\Delta G$ (respectively, 0.425 i.e. $92 \%, 0.463$ i.e. $89 \%$ and 0.441 i.e. $88 \%$ ) (Tables 1 and 2). The same comparisons with a FAC family structure are shown in Tables 1 and 2. Similarly, for the GS/SIB schemes, when heritability was increased from 0.4 to 1 , RAND, MC and MCAC had a lower $\triangle \mathrm{F}$ (respectively, 0.008 i.e. $29 \%, 0.002$ i.e. $14 \%$ and 0.005 i.e. $19 \%$ ) and a higher $\Delta \mathrm{G}$ (respectively, 0.143 i.e. $19 \%, 0.151$ i.e. $19 \%$ and 0.14 i.e. $18 \%$ ) (Tables 1 and 2). Similar results were obtained for the BLUP/CAND and the GS/ CAND schemes (Results not shown). Heritability had a similar effect on $\Delta \mathrm{F}$ and $\Delta \mathrm{G}$ when the intensity of selection was increased (Results not shown). Overall, increases in $\Delta \mathrm{G}$ due to increasing heritability were larger for schemes with a PAIR structure than for schemes with a FAC structure.

\section{Discussion}

This study was designed mainly for aquaculture breeding schemes. However, the conclusions can be extrapolated to any domesticated species. We examined the effect of non-random mating when genetic evaluation, information source, family structure and heritability varied. The approach maintained a constant intensity among candidates with truncation selection and examined differences in $\Delta \mathrm{F}$ and $\Delta \mathrm{G}$. The primary findings were that non-random mating designs had less impact on schemes with GS evaluation than on schemes with 
BLUP evaluation, and that this impact was greater for schemes with a PAIR structure than for schemes with a FAC structure and also greater for schemes with SIB information than for schemes with CAND information (Figure 1). We also compared MC with MCAC, a novel method proposed by Henryon et al. [2], but found that differences between these mating designs were small, with the balance of evidence pointing to $\mathrm{MC}$ as being more effective.

Among the different scenarios, the greatest effects of non-random mating, both for MC and MCAC, were a reduction of $\Delta \mathrm{F}$ rather than an increase of $\Delta \mathrm{G}$. Selection intensity $(i)$ was held constant, with truncation selection, and in such schemes the primary selective advantage is the individual's breeding value or its components (the EBV and prediction error) [16]. In such a system, $\Delta F$ increases with $i^{2}$ [17], thus one can expect that if $\Delta \mathrm{F}$ instead of $i$ had been held constant across schemes, then substantial benefits in $\Delta G$ would have emerged for the schemes with a low $\Delta \mathrm{F}$. This is because $i$ would need to be reduced in schemes with a greater $\Delta \mathrm{F}$, and thus $\Delta \mathrm{G}$ would decrease as it is directly proportional to $i$. This dependence of $\Delta \mathrm{F}$ on $i$ explains why more than two-fold increases in $\Delta \mathrm{F}$ were observed when the number of parents per sex was halved (cf. Tables 1,3 ). If the comparisons were performed with optimum contribution selection [3], then a broadly similar outcome may be anticipated, although some differences may occur as the selective advantage with optimum contributions is the estimated Mendelian sampling term [18] distinct from truncation selection where the selective advantage is the individual's breeding value or its components [16].

The outcomes of two of the dimensions of this study, GS versus BLUP and CAND versus SIB are driven by the increased accuracy of estimation of Mendelian sampling terms. Increased accuracy of Mendelian sampling terms in truncation selection reduces $\Delta \mathrm{F}$ primarily by reducing the importance of inherited selective advantages that are conferred by parents to offspring, as parental EBV have less weight in the selection decisions made on candidates. In the context of genomic evaluation and BLUP, the additional accuracy and impact on $\Delta \mathrm{F}$ have been highlighted by Daetwyler et al. [12]. The use of sib-testing is an extreme example, whereby all information for selection among the candidates is derived from their sibs; this sib information helps to increase the accuracy of the EBV of the sire and the dam, and through them the candidate, but provides no direct information on the candidate's Mendelian sampling term. Hence the benefit in $\Delta \mathrm{F}$ from using Cand versus SIB is smaller since the candidate's own performance does allow an estimate of the Mendelian term.

A heritability equal to 1 was included in this study to explore the impact of increased evaluation accuracy in schemes with SIB information. In schemes with CAND information, $h^{2}=1$ is the same as mass selection for both BLUP and GS and the impact of non-random mating on mass selection has been explored by Caballero et al. [6]. For schemes with SIB information, the contrast between BLUP and GS is strongest when $h^{2}=1$, since with BLUP the lack of information on Mendelian sampling terms of candidates remains, but with GS the accuracy of Mendelian sampling term estimates is expected to be high. This impact was observed, with substantial increases in $\Delta \mathrm{G}$, and with the greatest reductions in $\Delta \mathrm{F}$ occurring in schemes using genomic evaluations rather than BLUP, for which the reductions in $\Delta \mathrm{F}$ were relatively small. In the schemes using genomic selection, the number of animals tested in genomic evaluation was not large enough to achieve an accuracy of 1. However, it is plausible that such accuracy could be achieved in the future. In this case, the outcome would be different from those simulated in this study as there would be no need for the SIB testing, and then both CAND and SIB testing would proceed as if it was mass selection by truncation on a recorded phenotype with $h^{2}$ $=1$. In this case, there would still be potential to reduce $\Delta \mathrm{F}$ for the same $\Delta \mathrm{G}$ by using a FAC family structure, by using $\mathrm{MC}$ and other non-random mating schemes, and by using optimal contribution selection [19].

Schemes with a FAC structure are mating designs that do not depend on non-randomness, neither in relation to pedigree nor EBV. Such schemes consistently generated lower $\Delta \mathrm{F}$ compared to schemes with a PAIR structure (Table 1). These results are in agreement with almost all previous studies $[2,19]$ where lower $\Delta \mathrm{F}$ were generated in schemes with a FAC structure compared to schemes with a hierarchal family structure, and extend this result to schemes using genomic evaluation. One published exception to this finding of a benefit from a FAC family structure was in aquaculture [20], when restrictions were placed on family tanks (i.e. full-sib families) but the results of that study are ambiguous and need further clarification. The benefit of FAC family structures has been shown to result from the reduced variance in selective advantages by reducing the component of variance due to mates [21]. This is achieved by 'averaging' mate effects over a greater number of individuals. This is in contrast to PAIR family structures, where the selective advantages of the mates are completely confounded. Future selection decisions should reduce the deviation of long-term contributions of the ancestors from their optimal contributions. Relative to RAND mating, non-random mating designs substantially reduced $\Delta \mathrm{F}$ in schemes with a PAIR structure, but the differences were minor in the schemes with a FAC structure, most likely due to the improved structure already conferred by FAC. 
$\mathrm{MC}$ and MCAC both aim at improving family structure for selection but use different approaches: MCAC chooses mates to minimize the covariance of ancestral contributions, whereas MC minimizes the expected variance of contributions of the ancestors for a randomly chosen offspring. The latter is because the sum of squared contributions of the ancestors weighted by their within-family variance equals the diagonal of the relationship matrix, i.e. $1+\mathrm{F}$ of the offspring. Thus minimizing $\mathrm{F}$ by $\mathrm{MC}$ mating is approximately equivalent to minimising the sum of squares of contributions, and thus the variance of the contributions of an individual, since the mean contribution is not affected by the mating scheme. The justification for MCAC is that lower covariance among ancestral contributions allows future selection to shift individual contributions towards optimum values $[18,21]$, with less cost in inbreeding per unit change. In contrast, the smaller variance among ancestral contributions that is targeted by $\mathrm{MC}$ helps to minimize their changes when some offspring are selected. With the simulated schemes, the results suggest that minimizing the variance of contributions per offspring is more effective than minimizing the sum of covariances of ancestral contributions over all of the offspring. This could justify the use of MC instead of MCAC.

\section{Conclusions}

This study examined the benefits of non-random mating in simulated schemes with different scenarios. The conclusion is that non-random mating is more beneficial when the evaluation is based upon sib test records than candidate records. Changing the family structure from pair-wise to factorial was always beneficial in reducing rates of inbreeding but reduced the benefits from nonrandom mating per se. In this study, non-random mating was shown to be more beneficial with BLUP evaluation than with genomic selection.

\section{Acknowledgements}

This study was funded by grant 190442/S40 from the Research Council of Norway.

\section{Author details}

'Department of Animal and Aquacultural Sciences, Norwegian University of Life Science, Norwegian, P.O. Box 5003 N-1432 Ås, Norway. ${ }^{2}$ Nofima AS, Norwegian, P.O. Box 50101432 Ås, Norway. ${ }^{3}$ The Roslin Institute and Royal (Dick) School of Veterinary Studies, University of Edinburgh, Easter Bush Campus, Midlothian, EH25 9RG, Scotland, UK.

\section{Authors' contributions}

Kahsay G Nirea wrote the draft manuscript and ran the computer programs. Theo HE Meuwissen and Anna K Sonesson wrote simulation computer programs. Anna K Sonesson, John A Woolliams and Theo HE Meuwissen edited the drafted manuscript. Finally, all authors have approved the final manuscript for publication.

\section{Competing interests}

The authors declare that they have no competing interests.

Received: 24 October 2011 Accepted: 11 April 2012

Published: 11 April 2012

\section{References}

1. Sonesson AK, Meuwissen THE: Non-random mating for selection with restricted rates of inbreeding and overlapping generations. Genet Sel Evol 2002, 34:23-39.

2. Henryon M, Sørensen AC, Berg P: Mating animals by minimising the covariance between ancestral contributions generates less inbreeding without compromising genetic gain in breeding schemes with truncation selection. Animal 2009, 3:1339-1346.

3. Meuwissen THE: Maximizing the response of selection with a predefined rate of inbreeding. J Anim Sci 1997, 75:934-940.

4. Grundy B, Villanueva B, Woolliams JA: Dynamic selection procedures for constrained inbreeding and their consequences for pedigree development. Genet Res 1998, 72:159-168.

5. Sonesson AK, Meuwissen THE: Mating schemes for optimum contribution selection with constrained rates of inbreeding. Genet Sel Evol 2000, 32:231-248.

6. Caballero A, Santiago E, Toro MA: Systems of mating to reduce inbreeding in selected populations. Anim Sci 1996, 62:431-442.

7. Caballero A, Santiago E: Response to selection from new mutation and effective size of partially inbred populations. I. Theoretical results. Genet Res 1995, 66:213-225.

8. Woolliams JA: Modifications to MOET nucleus breeding schemes to improve rates of genetic progress and decrease rates of inbreeding in dairy cattle. Anim Prod 1989, 49:1-14.

9. Toro MA, Silio L, Rodrigañez J, Dobao MT: Inbreeding and family index selection for prolificacy in pigs. Anim Sci 1988, 46:79-85.

10. Santiago E, Caballero A: Effective size of populations under selection. Genetics 1995, 139:1013-1030.

11. Meuwissen THE, Hayes BJ, Goddard ME: Prediction of total genetic value using genome-wide dense marker maps. Genetics 2001, 157:1819-1829.

12. Daetwyler HD, Villanueva B, Bijma P, Woolliams JA: Inbreeding in genomewide selection. J Anim Breed Genet 2007, 124:369-376.

13. Sonesson AK, Meuwissen HE: Testing strategies for genomic selection in aquaculture breeding programs. Genet Sel Evol 2009, 41:37.

14. Henderson CR: Application of Linear Models in Animal Breeding Guelph: Canada Guelph University Press; 1984.

15. Nielsen HM, Sonesson AK, Yazdi H, Meuwissen THE: Comparison of accuracy of genome-wide and BLUP breeding value estimates in sib based aquaculture breeding schemes. Aquaculture 2009, 289:259-264.

16. Woolliams JA, Bijma P, Villanueva B: Expected genetic contributions and their impact on gene flow and genetic gain. Genetics 1999, 153:1009-1020.

17. Woolliams JA, Bijma P: Predicting rates of inbreeding in populations undergoing selection. Genetics 2000, 154:1851-1864.

18. Avendaño S, Woolliams JA, Villanueva B: Mendelian sampling terms as a selective advantage in optimum breeding schemes with restrictions on the rate of inbreeding. Genet Res 2004, 83:55-64.

19. Sorensen A, Berg P, Woolliams JA: The advantage of factorial mating under selection is uncovered by deterministically predicted rates of inbreeding. Genet Sel Evol 2005, 37:57-81.

20. Martinez V, Kause A, Mäntysaari E, Mäki-Tanila A: The use of alternative breeding schemes to enhance genetic improvement in rainbow trout (Oncorhynchus mykiss): I one-stage selection. Aquaculture 2006, 254:182-194.

21. Grundy B, Luo ZW, Villanueva B, Woolliams JA: The use of Mendelian indices to reduce the rate of inbreeding in selection programmes. $J$ Anim Breed Genet 1998, 115:39-51.

doi:10.1186/1297-9686-44-11

Cite this article as: Nirea et al: Effect of non-random mating on genomic and BLUP selection schemes. Genetics Selection Evolution 2012 44:11. 